\title{
微波促进范并咪唑氮杂环卡宾钯化合物催化的水相氭基化反应
}

\author{
蒋晓军 $a$ 由雅晴 $b$ 刘泽龙 ${ }^{b}$ 吕绪良 $*, a$ 涂涛*, $b$ \\ $\left({ }^{a}\right.$ 解放军理工大学野战工程学院 南京 210007) \\ ( $b$ 复旦大学化学系 上海 200433)
}

\begin{abstract}
摘要 报道了一种在微波条件下, 范并咪唑氮杂环卡宾钯化合物催化的芳基溴化物的氰化反应. 该反应以水作为溶剂, 无毒廉价的亚铁氰化钾为氰基源，对于含各种电子效应取代基团的底物均能顺利地进行反应并且在很短的时间内取得 优秀的产率, 充分表明了这一方法学具有较好的实用性和绿色经济性.

关键词 氰化反应; 氮杂环卡宾; 微波辅助; 钯催化; 水相
\end{abstract}

\section{Acenaphthoimidazole $\mathrm{N}$-Heterocyclic Carbene Palladium Complexes Catalyzed Cyanation Reactions in Aqueous Accelerated by Microwave Irradiation}

\author{
Jiang, Xiaojun $^{a} \quad$ Shen, Yajing $^{b} \quad$ Liu, Zelong $^{b} \quad$ Lü, Xuliang*a $\quad$ Tu, Tao ${ }^{*, b}$ \\ ( ${ }^{a}$ College of Field Engineering, PLA University of Science and Technology, Nanjing 210007) \\ ( ${ }^{b}$ Department of Chemistry, Fudan University, Shanghai 200433)
}

\begin{abstract}
In the presence of acenaphthoimidazole $N$-heterocyclic carbene palladium complexes, cyanation of aryl bromides by non-toxic and inexpensive potassium hexacyanoferrate (II) as cyanide source in water has been investigated under microwave conditions. Both electron-donating and electron-withdrawing groups attached to the substrates show unobvious effects on the transformation and produce the corresponding products in good to excellent yields within few minutes, which demonstrate the practicability and environmentally friendliness of the new developed protocol.
\end{abstract}

Keywords cyanation; $N$-heterocyclic carbene; microwave assistant; palladium; aqueous phase

含不同取代基团的苯甲腈类化合物是精细化工合 成中一类非常重要的合成子, 许多染料、除草剂、农药 以及天然药物中也都能发现这一重要的结构片段 ${ }^{[1]}$. 在 众多已知的芳香腈类化合物合成方法中 ${ }^{[2]}$, 过渡金属催 化的芳基卤化物的氰基化反应是一类非常高效实用的 合成方法. 对于这一方法学研究, 科学家们关注的焦点 主要是对低毒高效的氰基源开发方面. 1973 年 Takagi 课 题组 ${ }^{[3]}$ 第一次报道了以 $\mathrm{KCN}$ 为氰基源, 钯催化的芳基 卤代物的氰基化反应. 之后很多课题组都以 $\mathrm{KCN}$ 作为 氧基源, 考察了不同催化体系的催化效率 ${ }^{[4]}$, 并在此基 础上陆陆续续发展了 $\mathrm{NaCN}^{[5]} 、 \mathrm{Me}_{3} \mathrm{SiCN}^{[6]}$ 、 $n-\mathrm{Bu}_{3} \mathrm{SnCN}^{[7]}$ 和 $\mathrm{Zn}(\mathrm{CN})_{2}{ }^{[8]}$ 等新的氰基源应用于氰基化
反应. 但是这些氰基源大都有一些缺陷，限制了其进一 步的工业应用，如碱性氧化物一般都是剧毒物; 而 $\mathrm{Zn}(\mathrm{CN})_{2} 、 n-\mathrm{Bu}_{3} \mathrm{SnCN}$ 的使用会造成化学计量的重金属 盐的浪费; $\mathrm{Me}_{3} \mathrm{SiCN}$ 对水很敏感, 极易释放出剧毒气体 HCN, 造成安全隐患. 2004 年 Beller 课题组 ${ }^{[9]}$ 第一次使 用了 $\mathrm{K}_{4} \mathrm{Fe}(\mathrm{CN})_{6}$ 作为氰基源应用于这类反应, $\mathrm{K}_{4} \mathrm{Fe}(\mathrm{CN})_{6}$ 一方面便宜易得并且毒性较低, 后处理比较简单, 另一 方面，它包含的六个㲵基根均可参与反应，大大提高了 反应的原子经济性，因此进一步推动了氧化反应的发 展.

现今微波技术已经成为有机化学方法学中一种重 要的辅助手段 ${ }^{[10]}$, 特别是水具有较好的传质性质, 而且

\footnotetext{
* E-mail: xllu1957@126.com, taotu@fudan.edu.cn

Received June 24, 2015; revised July 9, 2015; published online August 17, 2015.

Project supported by the Research Fund of Doctoral Program, Ministry of Education (No. 20130071110032), the Shanghai Leading Academic Discipline Project (B108) and the Department of Chemistry Fudan University.

教育部博士点基金(No. 20130071110032)、上海市带头学科(No. B108)及复旦大学卓学资助项目.
} 
作为绿色经济溶剂无疑是有机方法学的首选, 因此近年 来微波促进的水相反应得到了很大的发展 ${ }^{[11]}$. 对于微波 促进的水相氰基化反应而言, 尽管已有一些报道的例 子, 但是仍然存在着对给电子基团和杂环底物不兼容以 及使用剧毒氰基源等问题, 因此探索一条更加绿色、低 毒、高效通用的途径仍然成为近年来该领域的研究热点.

氮杂环卡宾配体(NHCs)由于其低毒、对水热稳定以 及易于实现对电子和位阻效应调控等优点, 成为最近几 年来新型催化剂研究的重要对象 ${ }^{[12]}$. 由于增大的芳环 可以有效提高 NHCs 的 $\sigma$-给电子能力, 促进与底物的氧 化加成, 因此我们组设计合成的范并咪唑氮杂环卡宾配 体在大位阻 Suzuki 偶联反应、插羰反应以及 Buchwald 胺化等反应中均取得了非常不错的结果 ${ }^{[13]}$. 我们希望 进一步考察苊并咪唑氮杂环卡宾钯配合物作为催化剂 前体应用于氰基化反应的催化性能和底物普适, 进一步 拓展其应用范围.

\section{1 结果与讨论}

\section{1 实验讨论}

首先, 我们选择对溴苯乙酮作为标准底物考察了在 微波条件下范并咪唑氮杂环卡宾钯化合物 $\mathbf{1 a}$ 的催化性 能(表 1). 由于反应是在水相中进行的, 因此我们需加入 1 equiv. 四丁基溴化铵(TBAB)作为相转移催化剂以促进 底物催化剂的接触. 实验结果表明, 少量醇的存在是非 常必要的(表 1, Entry 1). 这点不难从催化剂的结构特征 来解释, 范并咪唑氮杂环卡宾钯化合物 $\mathbf{1}$ 并没有亲水基 团, 在水中的溶解度较差, 所以需要有机助溶剂增加其 溶解度从而来提高催化效率. 我们试了常用的有机助溶 剂甲醇、乙醇、异丙醇和乙腈(表 1, Entries 2 5), 在其 他条件不变的条件下异丙醇的加入可以得到最高的产 率 35\%, 这可能是因为异丙醇具有一定的还原性, 容易 将催化循环中的 $\operatorname{Pd}(\mathrm{II})$ 还原成 $\operatorname{Pd}(0)$, 从而完成催化循 环. 但是异丙醇的量过多对反应也是不利的, 当反应在 纯异丙醇溶剂中反应时, 只能得到极少量的产物(表 1 , Entries 11). 尽管经过上述条件篎选并没有得到一个比 较好的结果, 考虑到反应需要相转移催化剂来保证底物 和催化剂的接触, 所以对其加入量的调节应该是比较重 要的因素. 我们发现减少 TBAB 的量反而对反应有利, 但也只是得到 $42 \%$ 的产率, 升温至 $160{ }^{\circ} \mathrm{C}$ 也只是有微 小的提高(表 1, Entries 6 8). 令人高兴的是, 进一步降 低 TBAB 的量至 0.25 equiv. 可以得到几乎定量的产率(表 1, Entries 9 10). 这可能是由于过量的 TBAB 和异丙醇 的体系会降低 $\mathrm{K}_{4} \mathrm{Fe}(\mathrm{CN})_{6}$ 的溶解性. 除此之外对碱的篮 选表明, 除了 $\mathrm{NaF}$ 外其它有机、无机碱均不利于这个反 应(表 1, Entries 12 15). 然后我们测试了微波的作用,
实验结果表明在没有微波辅助的条件下只能得到 $83 \%$ 的产率，进一步证明了微波对反应的促进作用(表 1 , Entry 16). 因此得到最优的反应条件为: 在 $150 \mathrm{~W}$ 微波 功率辅助下, 向含 $0.5 \mathrm{~mol} \%$ 的催化剂 1a、 1 equiv. $\mathrm{NaF}$ 、 $0.5 \mathrm{mmol}$ 对溴苯乙酮及 $1 \mathrm{~mL}$ 无氧水的反应管中, 加入 0.25 equiv. TBAB 和 $0.2 \mathrm{~mL}$ 的异丙醇, 反应 $40 \mathrm{~min}$ 后可 以顺利得到相应的对氧基苯乙酮.

表 1 反应条件优化 ${ }^{a}$

Table 1 Optimization of the reaction conditions ${ }^{a}$
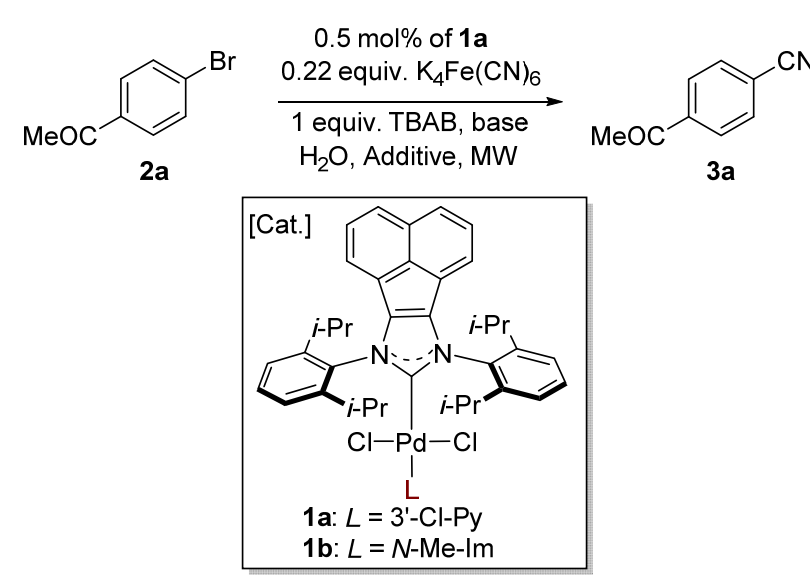

\begin{tabular}{lllcc}
\hline Entry & \multicolumn{1}{c}{ Base } & \multicolumn{1}{c}{ Additive $/ \mathrm{mL}$} & $T /{ }^{\circ} \mathrm{C}$ & Yield $^{b} \%$ \\
\hline 1 & $\mathrm{NaF}$ & - & 150 & Trace \\
2 & $\mathrm{NaF}$ & $0.2(i-\mathrm{PrOH})$ & 150 & 35 \\
3 & $\mathrm{NaF}$ & $0.2(\mathrm{EtOH})$ & 150 & 31 \\
4 & $\mathrm{NaF}$ & $0.2\left(\mathrm{CH}_{3} \mathrm{CN}\right)$ & 150 & Trace \\
5 & $\mathrm{NaF}$ & $0.2\left(\mathrm{CH}_{3} \mathrm{OH}\right)$ & 150 & Trace \\
$6^{c}$ & $\mathrm{NaF}$ & $0.2(i-\mathrm{PrOH})$ & 150 & Trace \\
$7^{d}$ & $\mathrm{NaF}$ & $0.2(i-\mathrm{PrOH})$ & 150 & 42 \\
$8^{d}$ & $\mathrm{NaF}$ & $0.2(i-\mathrm{PrOH})$ & 160 & 47 \\
$9^{e}$ & $\mathrm{NaF}$ & $0.2(i-\mathrm{PrOH})$ & 160 & 59 \\
$10^{f}$ & $\mathrm{NaF}$ & $0.2(i-\mathrm{PrOH})$ & 160 & 95 \\
$11^{f}$ & $\mathrm{NaF}^{f}$ & $1(i-\mathrm{PrOH})$ without water & 160 & Trace \\
$12^{f}$ & $\mathrm{Na}_{2} \mathrm{CO}$ & $0.2(i-\mathrm{PrOH})$ & 160 & 13 \\
$13^{f}$ & $\mathrm{~K}_{2} \mathrm{CO}$ & $0.2(i-\mathrm{PrOH})$ & 160 & Trace \\
$14^{f}$ & $\mathrm{NaOAc}_{3}$ & $0.2(i-\mathrm{PrOH})$ & 160 & NR \\
$15^{f}$ & $\mathrm{DBU}^{\prime}$ & $0.2(i-\mathrm{PrOH})$ & 160 & NR \\
$16^{f}$ & $\mathrm{NaF}$ & $0.2(i-\mathrm{PrOH})$ without MW & 160 & 83 \\
\hline
\end{tabular}

${ }^{a}$ Under the microwave irradiation, $0.5 \mathrm{mmol}$ scale with 1 equiv. of base, 0.22 equiv. of $\mathrm{K}_{4} \mathrm{Fe}(\mathrm{CN})_{6}, 1$ equiv. of TBAB and $1 \mathrm{~mL}$ of water for $20 \mathrm{~min} ;{ }^{b}$ isolated yield; ${ }^{c} 2$ equiv. of TBAB; ${ }^{d} 0.5$ equiv. of TBAB; ${ }^{e} 0.38$ equiv. of TBAB; ${ }^{f} 0.25$ equiv. of TBAB.

\section{2 普适性研究}

在得到最优条件之后, 我们对反应的普适性进行了 研究(表 2). 从表 2 可以看出, 无论是吸电子基团还是给 电子基团均可兼容于这个反应体系，并且取得非常优秀 的产率(表 2, Entries 1 4, 7). 这也验证了使用范并咪唑 氮杂环卡宾钯配合物的优越性. 接下来我们对底物的位 阻效应进行了进一步的探索，发现使用 $N$-甲基咪唑的 
催化剂 1b 时，间甲基溴苯得到最高的产率 97\%(表 2, Entry 5), 而位阻较大的邻甲基溴苯产率有所下降，只有 $71 \%$ (表 2, Entry 6), 由此可以看出这个体系对位阻效应 还是比较敏感的.

表 2 芳基溴化物的氭化反应 ${ }^{a}$

Table 2 The cyanation of aryl bromides

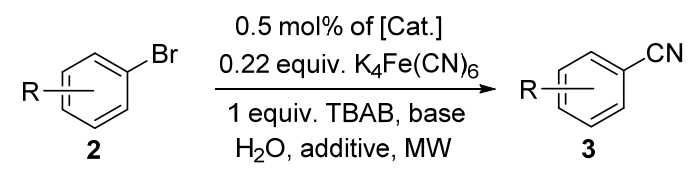

\begin{tabular}{|c|c|c|c|c|}
\hline Entry & $\mathrm{ArCN}$ & 3 & Base & Yield $^{b} / \%$ \\
\hline 1 & & $3 a$ & $\mathrm{NaF}$ & $95 \%^{c}$ \\
\hline 2 & & $3 b$ & $\mathrm{NaF}$ & $91^{e}$ \\
\hline 3 & & $3 c$ & $\mathrm{Na}_{2} \mathrm{CO}_{3}$ & 84 \\
\hline 4 & & 3d & $\mathrm{NaF}$ & $87^{e}$ \\
\hline 5 & & $3 e$ & $\mathrm{NaF}$ & $97^{e}$ \\
\hline 6 & & $3 f$ & $\mathrm{NaF}$ & $71^{e, f}$ \\
\hline 7 & & $3 g$ & $\mathrm{NaF}$ & 68 \\
\hline
\end{tabular}

${ }^{a}$ Under the microwave irradiation, $0.5 \mathrm{mmol}$ scale with 0.25 equiv. TBAB, 1 equiv. base, 0.25 equiv. $\mathrm{K}_{4} \mathrm{Fe}(\mathrm{CN})_{6}, 1 \mathrm{~mL} \mathrm{H}_{2} \mathrm{O}+0.2 \mathrm{~mL} i$-PrOH, 1 equiv. $\mathrm{NaI}$, $160{ }^{\circ} \mathrm{C}, 40 \mathrm{~min} ;{ }^{b}$ isolated yield; ${ }^{c} 20 \mathrm{~min}$ without $\mathrm{NaI} ;{ }^{d}$ with 0.5 equiv. TBAB; ${ }^{e}$ with $1 \mathrm{~mL} \mathrm{H} 2 \mathrm{O}+0.4 \mathrm{~mL} i$-PrOH$;{ }^{f}$ with $\mathbf{1 b}$ as catalyst.

\section{2 结论}

实现了范并咪唑氮杂环卡宾钯化合物催化下微波 促进的水相氰基化反应. 经过对条件的仔细笁选, 考察 了相转移催化剂 TBAB 的量、温度、有机助溶剂以及碱 对反应的影响, 得到了反应的最优条件. 随后对底物的 普适性进行了研究, 不论底物是否连有吸电子基团、给 电子基团, 或者是大位阻的芳基溴化物, 都可以良好的 产率得到相应的产物. 由于该反应使用的是低毒的氰基 源, 而且是以水作为溶剂的, 因此为高效合成腈类化合 物提供了更为绿色经济的方法.

\section{3 实验部分}

\section{1 仪器与试剂}

所有的反应均在微波反应器 CEM Discover microwave instrument 中进行. ${ }^{1} \mathrm{H}$ NMR $(400 \mathrm{MHz})$ 和 ${ }^{13} \mathrm{C} \mathrm{NMR}$ (100 MHz)检测采用 AVANCE III 400 核磁共振仪; MS (ESI)检测采用 $1100 \mathrm{LC} / \mathrm{MSD}$ SL 质谱仪(美国 Agilent 公司); HRMS (ESI)检测采用 APEX III ${ }^{\mathrm{TM}}$ ESI-FTICRMS 质谱仪(美国 Bruker Daltonics 公司); 熔点检测采用 SGW X-4 显微熔点仪(上海精密科学仪器有限公司). 无 氧水由实验室抽真空脱气所制; 其它试剂和药品全部从 市场直接购买.

\section{2 实验方法}

范并咪唑氮杂环卡宾钯化合物合成的一般方法 ${ }^{[13 c]}$ : 在氮气保护下，向 Schlenk 瓶中加入 $\mathrm{PdCl}_{2}(0.088 \mathrm{~g}, 0.5$ $\mathrm{mmol}), N, N^{\prime}-(2,6$-二异丙基苯基)-范并咪唑盐酸盐( 0.55 $\mathrm{mmol}), \mathrm{K}_{2} \mathrm{CO}_{3}(0.345 \mathrm{~g}, 5.0 \mathrm{mmol}), 3$-氯吡啶 $(2.0 \mathrm{~mL})$. 反应混合物于 $90{ }^{\circ} \mathrm{C}$ 下剧烈搅拌 $24 \mathrm{~h}$ 后冷至室温. 加入 二氯甲烷稀释, 经硅藻土过滤后蒸去溶剂, 减压下除去 3-氯吡啶. 经氯仿/石油梄重结晶后得到产品 $0.305 \mathrm{~g}$ $(76 \%)$.

三氯吡啶稳定的范并咪唑氮杂环卡宾钯化合物 (1a) ${ }^{[13 \mathrm{c}]}$ : 黄色固体. ${ }^{1} \mathrm{H} \mathrm{NMR}\left(\mathrm{CDCl}_{3}, 400 \mathrm{MHz}, 298 \mathrm{~K}\right) \delta$ : $8.68(\mathrm{~d}, J=2.0 \mathrm{~Hz}, 1 \mathrm{H}), 8.61$ (d, $J=5.2 \mathrm{~Hz}, 1 \mathrm{H}), 7.70$ (d, $J=8.4 \mathrm{~Hz}, 2 \mathrm{H}), 7.64(\mathrm{t}, J=8.0 \mathrm{~Hz}, 2 \mathrm{H}), 7.57$ (d, $J=8.0$ $\mathrm{Hz}, 1 \mathrm{H}), 7.48$ (d, $J=8.0 \mathrm{~Hz}, 4 \mathrm{H}), 7.34$ (t, $J=7.6 \mathrm{~Hz}, 2 \mathrm{H})$, $7.10(\mathrm{dd}, J=8.0 \mathrm{~Hz}, 5.6 \mathrm{~Hz}, 1 \mathrm{H}), 6.80(\mathrm{~d}, J=7.32 \mathrm{~Hz}$, 2H), $3.45 \sim 3.35(\mathrm{~m}, 4 \mathrm{H}), 1.46(\mathrm{~d}, J=6.4 \mathrm{~Hz}, 12 \mathrm{H}), 0.92$ $(\mathrm{d}, J=6.4 \mathrm{~Hz}, 12 \mathrm{H}) ;{ }^{13} \mathrm{C} \mathrm{NMR}\left(\mathrm{CDCl}_{3}, 100 \mathrm{MHz}, 298 \mathrm{~K}\right)$ $\delta: 159.07,150.49,149.48,147.21,140.39,137.37,133.79$, $131.78,130.65,129.51,129.05,128.06,127.21,126.02$, 124.71, 124.24, 122.13, 28.86, 25.76, 24.24. HRMS (ESI) calcd for $\mathrm{C}_{42} \mathrm{H}_{44} \mathrm{Cl}_{3} \mathrm{~N}_{3} \mathrm{Pd}[\mathrm{M}-\mathrm{Cl}-\mathrm{Py}-2 \mathrm{Cl}]^{+}$617.2226, found 617.2163 .

$N$-甲基咪唑稳定的范并咪唑氮杂环卡宾钯化合物 (1b) ${ }^{[13 \mathrm{c}]}$ : 黄色固体. ${ }^{1} \mathrm{H} \mathrm{NMR}\left(\mathrm{CDCl}_{3}, 400 \mathrm{MHz}, 298 \mathrm{~K}\right) \delta$ : 7.76 (s, 1H), 7.67 (d, $J=7.6 \mathrm{~Hz}, 2 \mathrm{H}), 7.58$ (d, $J=6.8 \mathrm{~Hz}$, 2H), $7.45(\mathrm{~d}, J=7.2 \mathrm{~Hz}, 4 \mathrm{H}), 7.35 \sim 7.05(\mathrm{~m}, 2 \mathrm{H}), 7.26(\mathrm{~s}$, $1 \mathrm{H}), 6.75(\mathrm{~d}, J=6.0 \mathrm{~Hz}, 2 \mathrm{H}), 6.53(\mathrm{~s}, 1 \mathrm{H}), 3.42$ (s, 7H), $1.45(\mathrm{~d}, J=5.2 \mathrm{~Hz}, 12 \mathrm{H}), 0.90(\mathrm{~d}, J=6.0 \mathrm{~Hz}, 12 \mathrm{H}) ;{ }^{13} \mathrm{C}$ NMR $\left(\mathrm{CDCl}_{3}, 100 \mathrm{MHz}, 298 \mathrm{~K}\right) \delta: 162.5,147.3,140.3$, $138.4,134.1,130.4,129.5,129.1,128.5,127.8,127.2$, 126.2, 124.6, 121.9, 119.0, 33.9, 28.8, 25.7, 24.3.

水相氰基化反应的一般步骤: 按所述条件将催化剂 $(0.5 \mathrm{~mol} \%) 、 \mathrm{TBAB}$ 、亚铁氭化钾、 $\mathrm{NaF}$ 或 $\mathrm{Na}_{2} \mathrm{CO}_{3}$ (1 
equiv.)、芳基卤化物(0.5 mmol)加入微波反应管中，抽真 空充氮气 3 次后加入无氧水 $1 \mathrm{~mL}$, 异丙醇 $(0.2 \sim 0.4$ $\mathrm{mL}$ ), 加入磁子密封后置于微波反应器中, 于 $160{ }^{\circ} \mathrm{C}$ 下 反应 $20 \sim 40 \mathrm{~min}$ (设定功率 $150 \mathrm{~W}$ ). 冷却取出后乙酸乙 酯萃取, 合并有机层后硫酸钠干燥, 柱层析得到产品.

对氰基苯乙酮(3a) ${ }^{[14]}$ : m.p. 58 59 ${ }^{\circ} \mathrm{C}$ (lit. ${ }^{[14]}$ 58 $\left.60{ }^{\circ} \mathrm{C}\right) ;{ }^{1} \mathrm{H}$ NMR $\left(400 \mathrm{MHz}, \mathrm{CDCl}_{3}, 298 \mathrm{~K}\right) \delta: 8.04$ (d, $J=$ $8.4 \mathrm{~Hz}, 2 \mathrm{H}), 7.78$ (d, $J=8.8 \mathrm{~Hz}, 2 \mathrm{H}), 2.65$ (s, 3H); GC-MS $\mathrm{m} / z: 145[\mathrm{M}]^{+}, 130[\mathrm{M}-\mathrm{Me}]^{+}, 102[\mathrm{M}-\mathrm{COMe}]^{+}$.

4-苯基苯甲腈 $(3 b)^{[15]}$ : m.p. 83 $85{ }^{\circ} \mathrm{C}$ (lit. ${ }^{[15]} 85 \sim$ $\left.86{ }^{\circ} \mathrm{C}\right) ;{ }^{1} \mathrm{H} \mathrm{NMR}\left(400 \mathrm{MHz}, \mathrm{CDCl}_{3}, 298 \mathrm{~K}\right) \delta: 7.73$ (d, $J=$ $8.4 \mathrm{~Hz}, 2 \mathrm{H}), 7.69$ (d, $J=8.4 \mathrm{~Hz}, 2 \mathrm{H}), 7.59$ (d, $J=7.2 \mathrm{~Hz}$, 2H), 7.49 (t, $J=7.2 \mathrm{~Hz}, 2 \mathrm{H}), 7.43$ (t, $J=7.2 \mathrm{~Hz}, 1 \mathrm{H})$; GC-MS $m / z: 179[\mathrm{M}]^{+}, 151[\mathrm{M}-\mathrm{CN}]^{+}$.

4-甲氧基苯甲腈 $(3 c)^{[14]}$ : m.p. 59 $61{ }^{\circ} \mathrm{C}$ (lit. ${ }^{[14]} 60 \sim$ $\left.61{ }^{\circ} \mathrm{C}\right) ;{ }^{1} \mathrm{H}$ NMR $\left(400 \mathrm{MHz}, \mathrm{CDCl}_{3}, 298 \mathrm{~K}\right) \delta: 7.59(\mathrm{dt}$, $J=8.8,2.4 \mathrm{~Hz}, 2 \mathrm{H}), 6.95$ (dt, $J=9.2,2.4 \mathrm{~Hz}, 2 \mathrm{H}), 3.86$ (s, 3H); GC-MS m/z: 133 [M] $]^{+}, 118,103,90$.

4-甲基苯甲腈 $(3 d){ }^{[16]}$ : 淡黄色液体. ${ }^{1} \mathrm{H}$ NMR (400 $\left.\mathrm{MHz}, \mathrm{CDCl}_{3}, 298 \mathrm{~K}\right) \delta$ : 7.53 (d, $\left.J=8.0 \mathrm{~Hz}, 2 \mathrm{H}\right), 7.26$ (d, $J=8.0 \mathrm{~Hz}, 2 \mathrm{H}), 2.42(\mathrm{~s}, 3 \mathrm{H})$; GC-MS $m / z: 117[\mathrm{M}]^{+}, 90$.

3-甲基苯甲腈 $(3 \mathbf{e})^{[17]}$ : 淡黄色液体. ${ }^{1} \mathrm{H}$ NMR (400 $\left.\mathrm{MHz}, \mathrm{CDCl}_{3}, 298 \mathrm{~K}\right) \delta: 7.44 \sim 7.37(\mathrm{~m}, 3 \mathrm{H}), 7.36 \sim 7.30$ (m, 1H), 2.37 (s, 3H); GC-MS m/z: $117[\mathrm{M}]^{+}, 90$.

2-甲基苯甲腈 $(\mathbf{3 f})^{[16]}$ : 无色液体. ${ }^{1} \mathrm{H}$ NMR (400 $\left.\mathrm{MHz}, \mathrm{CDCl}_{3}, 298 \mathrm{~K}\right) \delta: 7.56(\mathrm{~d}, J=7.6 \mathrm{~Hz}, 1 \mathrm{H}), 7.46$ (t, $J=7.6 \mathrm{~Hz}, 1 \mathrm{H}), 7.31 \sim 7.22(\mathrm{~m}, 2 \mathrm{H}), 2.52(\mathrm{~s}, 3 \mathrm{H})$; GC-MS $m / z: 117[\mathrm{M}]^{+}, 90$.

4-苯甲酮基苯甲腈 $(\mathbf{3 g})^{[18]}$ : m.p. $112 \sim 113{ }^{\circ} \mathrm{C}$ (lit. ${ }^{[19]}$ $\left.114 \sim 116{ }^{\circ} \mathrm{C}\right) ;{ }^{1} \mathrm{H}$ NMR (400 MHz, $\left.\mathrm{CDCl}_{3}, 298 \mathrm{~K}\right) \delta$ : $7.89 \sim 7.87(\mathrm{~m}, 2 \mathrm{H}), 7.81 \sim 7.78(\mathrm{~m}, 4 \mathrm{H}), 7.66 \sim 7.62 \mathrm{~m}$, 1H), $7.54 \sim 7.50(\mathrm{~m}, 2 \mathrm{H})$; GC-MS m/z: $207[\mathrm{M}]^{+}, 105$.

辅助材料(Supporting Information) 所合成目标化合 物的 NMR 图谱. 这些材料可以免费从本刊网站(http:// sioc-journal.cn/)上下载

\section{References}

[1] Kleemann, A.; Engel, J.; Kutscher, B.; Reichert, D. Pharmaceutical Substances: Syntheses, Patents, Applications, 4th ed., Georg Thieme, Stuttgart, 2001.

[2] (a) Larock, R. C. Comprehensive Organic Transformations, VCH, New York, 1989, p. 819.

(b) Grundmann, C. In Houben-Weyl: Methoden der Organischen Chemie, 4th ed., Ed.: Falbe, J., Georg Thieme Verlag, Stuttgart, 1985, Vol. E5, p. 1313.

(c) Hagedorn, F.; Gelbke, H.-P. In Ullmanns Encyklopadie der Technischen Chemie, 4th ed., Eds.: Bartholomé, E.; Biekert, E.;
Hellmann, H.; Ley, H.; Weigert, W. M.; Weise, E., Verlag Chemie, Weinheim, 1979, Vol. 17, p. 333.

(d) Ferri, C. Reaktionen der Organischen Chemie, Georg Thieme Verlag, Stuttgart, 1978, p. 571.

(e) Kurtz, P. In Houben Weyl: Methoden Der Organischen Chemie, 4th ed., Ed.: Muller, E., Georg Thieme Verlag, Stuttgart, 1952, Vol. 8, p. 265.

[3] Takagi, K.; Okamoto, T.; Sakakibara, Y.; Oka, S. Chem. Lett. 1973, 471.

[4] (a) Sekiya, A.; Ishikawa, N. Chem. Lett. 1975, 277.

(b) Takagi, K.; Okamoto, T.; Sakakibara, Y.; Ohno, A.; Oka, S.; Hayama, N. Bull. Chem. Soc. Jpn. 1975, 48, 3298.

(c) Akita, Y.; Shimazaki, M.; Ohta, A. Synthesis. 1981, 974.

(d) Prochazka, M.; Siroky, M. Collect. Czech. Chem. Commun. 1983, 48, 1765.

(e) Sato, N.; Suzuki, M. J. Heterocycl. Chem. 1987, 24, 1371.

(f) Tanji, K.; Higashino, T. Heterocycles 1990, 30, 435.

(g) Takagi, K.; Sasaki, K.; Sakakibara, Y. Bull. Chem. Soc. Jpn. 1991, 64, 1118

[5] Dalton, J. R.; Regen, S. L. J. Org. Chem. 1979, 44, 4443.

[6] Chatani, N.; Hanafusa, T. J. Org. Chem. 1986, 51, 4714.

[7] Nair, V.; Purdy, D. F.; Sells, T. B. J. Chem. Soc., Chem. Commun. 1989, 878 .

[8] (a) Tschaen, D. M.; Desmond, R.; King, A. O.; Fortin, M. C.; Pipik, B.; King, S.; Verhoeven, T. R. Synth. Commun. 1994, 24, 887.

(b) Gundersen, L. L. Acta Chem. Scand. 1996, 50, 58.

[9] (a) Schareina, T.; Zapf, A.; Beller, M. Chem. Commun. 2004, 1388. (b) Merz, V.; Weith, W. Chem. Ber. 1877, 10, 746.

[10] Gedye, R.; Smith, F.; Westaway, K.; Ali, H.; Baldisera, L.; Laberge, L.; Rousell, J. Tetrahedron Lett. 1986, 27, 279.

[11] (a) Li, L.; Pan, Z.; Duan, X.; Liang, Y. Synlett. 2006, 2094.

(b) Velmathi, S.; Leadbeate, N. Tetrahedron Lett. 2008, 49, 4693.

(c) Chen, G.; Weng, J.; Zheng, Z.; Zhu, X.; Cai, Y.; Cai, J.; Wan, Y. Eur. J. Org. Chem. 2008, 22, 3524.

(d) Ren, Y. L.; Wang, W.; Zhao, S.; Tian, X.; Wang, J.; Yin, W.; Cheng, L. Tetrahedron Lett. 2009, 50, 4595 .

(e) Fang, K.; Wen, W.; Chen, L.; Peng, L. Chin. J. Org. Chem. 2013, 33, 2559 (in Chinese).

(柯方, 吴雯, 林晨, 李鹏, 有机化学, 2013, 33, 2559.)

[12] Matthew, N.; Hopkinson, C. R.; Michael, S.; Glorius, F. Nat. Chem. 2014, 510, 485 .

[13] (a) Tu, T.; Feng, X.; Wang, Z.; Liu, X. Dalton Trans. 2010, 39, 10598.

(b) Tu, T.; Mao, H.; Herbert, C.; Xu, M.; Dötzb, K. H. Chem. Commun. 2010, 46, 7796.

(c) Tu, T.; Fang, W.; Jiang, J. Chem. Commun. 2011, 47, 12358.

(d) Tu, T.; Sun, Z.; Fang, W.; Xu, M.; Zhou, Y. Org. Lett. 2012, 14, 4250.

(e) Fang, W.; Deng, Q.; Xu, M.; Tu, T. Org. Lett. 2013, 15, 3678.

(f) Jiang, J.; Zhu, H.; Shen, Y.; Tu, T. Org. Chem. Front. 2014, 1, 1172.

[14] Alagiri, K.; Prabhu, K. R. Tetrahedron 2011, 67, 8544.

[15] Zhou, W.; Wang, K.; Wang, J. Adv. Synth. Catal. 2009, 351, 1378.

[16] Grossman, O.; Gelman, D. Org. Lett. 2006, 8, 1189.

[17] Mino, T.; Koizumi, T.; Shibuya, M.; Hirai, K.; Sakamoto, M.; Fujita, T. Heterocycles 2011, 83, 163.

[18] Karthikeyan, J.; Parthasarathy, K.; Cheng, C. Chem. Commun. 2011, 47, 10461.

Qin, W.; Yasuike, S.; Kakusawa, N.; Kurita, J. J. Organomet. Chem. 2008, 693, 2949.

(Li, L.; Fan, Y.) 